\title{
Traveling-Wave Solution of Modified Liouville Equation by Means of Modified Simple Equation Method
}

\author{
Md. Abdus Salam \\ Department of Computer Science and Engineering, Prime University, Dhaka-1216, Bangladesh \\ Correspondence should be addressed to Md. Abdus Salam, salam.a.math03@gmail.com \\ Received 2 August 2012; Accepted 21 August 2012 \\ Academic Editors: J. Kou and S. Sture \\ Copyright (c) 2012 Md. Abdus Salam. This is an open access article distributed under the Creative \\ Commons Attribution License, which permits unrestricted use, distribution, and reproduction in \\ any medium, provided the original work is properly cited. \\ We construct the traveling wave solutions involving parameters of modified Liouville equation \\ by using a new approach, namely the modified simple equation method. The proposed method is \\ direct, concise, and elementary and can be used for many other nonlinear evolution equations.
}

\section{Introduction}

The investigation of the traveling-wave solutions of nonlinear partial differential equations plays an important role in the study of nonlinear physical phenomena. Several direct methods for finding the explicit traveling-wave solutions to nonlinear partial differential equations have been proposed, such as the tanh-function method and its various extension [1], the Jacobi elliptic function expansion method [2], the homogeneous balance method $[3,4]$, the F-expansion method and its extension [5], the variational iteration method [6], $\left(G^{\prime} / G\right)$-expansion method [7], and so on. More recently, a new method, named modified simple equation method $[8,9]$, has been proposed to construct more explicit traveling-wave solutions of modified Liouville equation.

\section{Description of the Modified Simple Equation Method}

Suppose that a nonlinear equation, say in two independent variables $x$ and $t$ is given by

$$
P\left(u, u_{t}, u_{x}, u_{t t}, u_{x t}, u_{x x}, \ldots\right)=0
$$


where $u=u(x, t)$ is an unknown function, $P$ is a polynomial in $u=u(x, t)$ and its various partial derivatives, in which the highest-order derivatives and nonlinear terms are involved. In the following, the main steps of the modified simple equation method are given.

Step 1. The traveling-wave variable

$$
u(x, t)=u(\xi), \quad \text { where } \xi=A x+B t
$$

permits us reducing (2.1) to an ODE for $u=u(\xi)$ in the form

$$
P\left(u,-V u^{\prime}, u^{\prime}, V^{2} u^{\prime \prime},-V u^{\prime \prime}, u^{\prime \prime}, \ldots\right)=0 \text {. }
$$

Step 2. Suppose that the solution of ODE (2.1) can be expressed by a polynomial in $\left(\psi^{\prime} / \psi\right)$ as follows:

$$
u(\xi)=\sum_{i=0}^{n} \alpha_{i}\left(\frac{\psi^{\prime}}{\psi}\right)^{i}
$$

where $\alpha_{i}$ are arbitrary constants to be determined such that $\alpha_{n} \neq 0$, while $\psi(\xi)$ is an unknown function to be determined later.

Step 3. We determine the positive integer $n$ by considering the homogeneous balance between the highest order derivatives and nonlinear terms appearing in ODE (2.3).

Step 4. We substitute (2.4) into (2.3), we calculate all the necessary derivatives $u^{\prime}, u^{\prime \prime}, \ldots$, and then we account the function $\psi(\xi)$. As a result of this substitution, we get a polynomial of $\psi^{\prime} / \psi$ and its derivatives. In this polynomial, we equate with zero all the coefficients of it. This operation yields a system of equations which can be solved to find $\alpha_{i}$ and $\psi(\xi)$. Consequently, we can get the exact solution of (2.1).

\section{Application of the Method}

In this section, we would like to use our method to obtain new and more general exact traveling wave solutions of the modified Liouville equation

$$
w_{t t}=a^{2} w_{x x}+b e^{\beta w}
$$

where $a, b$, and $\beta$ are arbitrary constants.

Suppose $e^{\beta w}=u(x, t)$, where the traveling-wave transformation is

$$
u(x, t)=u(\xi), \quad \xi=A x+B t
$$

By using the traveling-wave variable (3.2), (3.1) is converted into an ODE for $u=u(\xi)$

$$
u u^{\prime \prime}-u^{\prime 2}+k u^{3}=0, \quad \text { where } k=\frac{b \beta}{a^{2} A^{2}-B^{2}} .
$$


Suppose that the solution of the ODE (3.3) can be expressed by a polynomial in $\left(\psi^{\prime} / \psi\right)$ as follows:

$$
u(\xi)=\sum_{i=0}^{n} \alpha_{i}\left(\frac{\psi^{\prime}}{\psi}\right)^{i}
$$

where $\alpha_{i}$ are arbitrary constants provided $\alpha_{n} \neq 0$.

Considering the homogeneous balance between the highest order derivatives and the nonlinear terms in (3.3), we get $n=2$ and hence the solution takes the following form:

$$
u(\xi)=\alpha_{0}+\alpha_{1}\left(\frac{\psi^{\prime}}{\psi}\right)+\alpha_{2}\left(\frac{\psi^{\prime}}{\psi}\right)^{2}
$$

where $\alpha_{2} \neq 0$. On substituting (3.5) into the ODE (3.3) and equating all the coefficients of $\psi^{-1}, \psi^{-2}, \psi^{-3}, \psi^{-4}, \psi^{-5}, \psi^{-6}$ to zero, we, respectively, obtain

$$
\begin{gathered}
k \alpha_{0}^{3}=0, \\
3 k \alpha_{0}^{2} \alpha_{1} \psi^{\prime}+\alpha_{0} \alpha_{1} \psi^{\prime \prime \prime}=0, \\
2 \alpha_{0} \alpha_{2} \psi^{\prime \prime 2}-\alpha_{1}^{2} \psi^{\prime \prime 2}-3 \alpha_{0} \alpha_{1} \psi^{\prime \prime} \psi^{\prime}+3 k \alpha_{0}^{2} \alpha_{2} \psi^{\prime 2}+3 k \alpha_{0} \alpha_{1}^{2} \psi^{\prime 2} \\
+2 \alpha_{0} \alpha_{2} \psi^{\prime} \psi^{\prime \prime \prime}+\alpha_{1}^{2} \psi^{\prime} \psi^{\prime \prime \prime}=0, \\
k \alpha_{1}^{3} \psi^{\prime 3}+2 \alpha_{0} \alpha_{1} \psi^{\prime 3}-2 \alpha_{1} \alpha_{2} \psi^{\prime} \psi^{\prime \prime 2}-\alpha_{1}^{2} \psi^{\prime 2} \psi^{\prime \prime}+3 \alpha_{1} \alpha_{2} \psi^{\prime 2} \psi^{\prime \prime \prime} \\
-10 \alpha_{0} \alpha_{2} \psi^{\prime 2} \psi^{\prime \prime}+6 k \alpha_{0} \alpha_{1} \alpha_{2} \psi^{\prime 3}=0, \\
-2 \alpha_{2}^{2} \psi^{\prime 2} \psi^{\prime \prime 2}+2 \alpha_{2}^{2} \psi^{\prime 3} \psi^{\prime \prime \prime}+\alpha_{1}^{2} \psi^{\prime 4}+3 k \alpha_{0} \alpha_{2}^{2} \psi^{\prime 4}+3 k \alpha_{1}^{2} \alpha_{2} \psi^{\prime 4} \\
+6 \alpha_{0} \alpha_{2} \psi^{\prime 4}-5 \alpha_{1} \alpha_{2} \psi^{\prime 3} \psi^{\prime \prime}=0, \\
3 k \alpha_{1} \alpha_{2}^{2} \psi^{\prime 5}-2 \alpha_{2}^{2} \psi^{\prime 4} \psi^{\prime \prime}+4 \alpha_{1} \alpha_{2} \psi^{15}=0, \\
k \alpha_{2}^{3} \psi^{\prime 6}+2 \alpha_{2}^{2} \psi^{\prime 6}=0 .
\end{gathered}
$$

Equations (3.6), (3.8), and (3.12) give $\alpha_{0}=0, \alpha_{1}=0, \alpha_{2}=-2 / k\left(\psi^{\prime} \neq 0\right.$, otherwise it is the trivial case); those satisfy (3.7) and (3.9), and (3.10), (3.11), respectively, yields

$$
\begin{gathered}
\psi^{\prime \prime 2}-\psi^{\prime} \psi^{\prime \prime \prime}=0, \\
\psi^{\prime \prime}=0 .
\end{gathered}
$$

Equation (3.14) gives $\psi^{\prime \prime}=0$. Integrating $\psi^{\prime \prime}=0$ with respect to $\xi$, we get $\psi=C_{1}+C_{2} \xi$ and the solution of ODE (3.3) takes the following form:

$$
u(\xi)=\frac{-2}{k}\left(\frac{C_{1}}{C_{1}+C_{2} \xi}\right)^{2}=\frac{2\left(B^{2}-a^{2} A^{2}\right)}{b \beta}\left(\frac{C}{A x+B t+C}\right)^{2} \text {, where } C=\frac{C_{1}}{C_{2}}
$$


And finally the traveling-wave solution of (3.1) is

$$
w=\frac{1}{\beta} \ln \left[\frac{2\left(B^{2}-a^{2} A^{2}\right)}{b \beta}\left(\frac{C}{A x+B t+C}\right)^{2}\right]
$$

\section{Conclusion}

On comparing this method with the other methods via the tanh-function method, homogeneous balance method, and the $\left(G^{\prime} / G\right)$-expansion method used in $[1,4,7]$, we see that the modified simple equation method is much more simpler than these methods because these methods have used the computer programs, while the modified simple equation method has not used these programs. Also we deduce that the modified simple equation method is effective and standard which allows us to solve complicated nonlinear evolution equations in the mathematical physics.

\section{References}

[1] E. Fan, "Extended tanh-function method and its applications to nonlinear equations," Physics Letters A, vol. 277, no. 4-5, pp. 212-218, 2000

[2] Z. Yan, "Abundant families of Jacobi elliptic function solutions of the $(2+1)$-dimensional integrable Davey-Stewartson-type equation via a new method," Chaos, Solitons and Fractals, vol. 18, no. 2, pp. 299-309, 2003.

[3] M. Wang, "Exact solutions for a compound KdV-Burgers equation," Physics Letters A, vol. 213, no. 5-6, pp. 279-287, 1996.

[4] M. Wang, Y. Zhou, and Z. Li, "Application of a homogeneous balance method to exact solutions of nonlinear equations in mathematical physics," Physics Letters A, vol. 216, no. 1-5, pp. 67-75, 1996.

[5] M. Wang and Y. Zhou, "The periodic wave solutions for the Klein-Gordon-Schrödinger equations," Physics Letters A, vol. 318, no. 1-2, pp. 84-92, 2003.

[6] N. A. Kudryashov, "Exact solutions of the generalized Kuramoto-Sivashinsky equation," Physics Letters A, vol. 147, no. 5-6, pp. 287-291, 1990

[7] M. Wang, X. Li, and J. Zhang, "The $\left(G^{\prime} / G\right)$-expansion method and travelling wave solutions of nonlinear evolution equations in mathematical physics," Physics Letters A, vol. 372, no. 4, pp. 417-423, 2008.

[8] A. J. M. Jawad, M. D. Petković, and A. Biswas, "Modified simple equation method for nonlinear evolution equations," Applied Mathematics and Computation, vol. 217, no. 2, pp. 869-877, 2010.

[9] E. M. E. Zayed and S. A. Hoda Ibrahim, "Exact solutions of nonlinear evolution equations in mathematical physics using the modified simple equation method," Chinese Physics Letters, vol. 29, no. 6, Article ID 060201, 2012. 


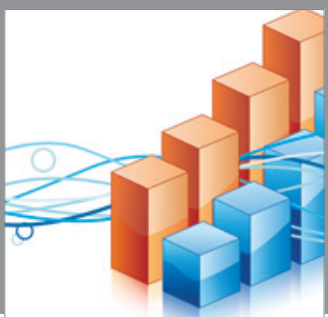

Advances in

Operations Research

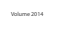

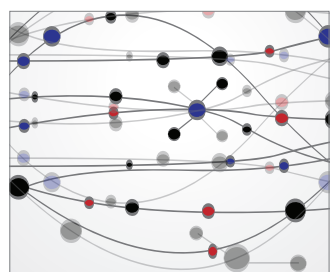

\section{The Scientific} World Journal
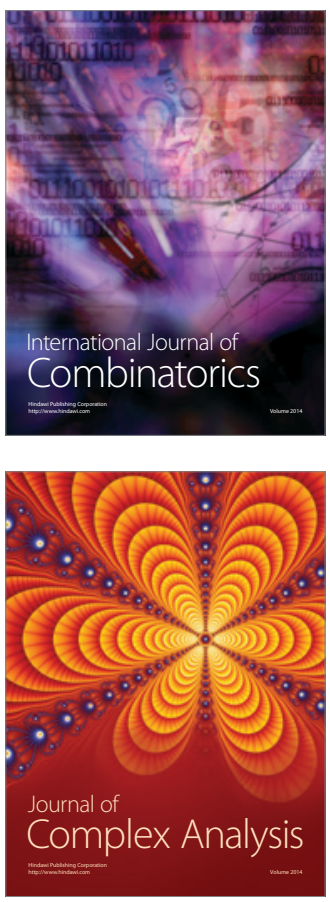

International Journal of

Mathematics and

Mathematical

Sciences
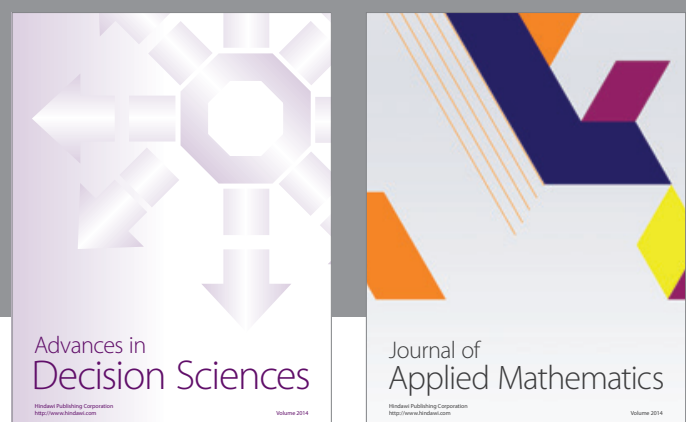

Journal of

Applied Mathematics
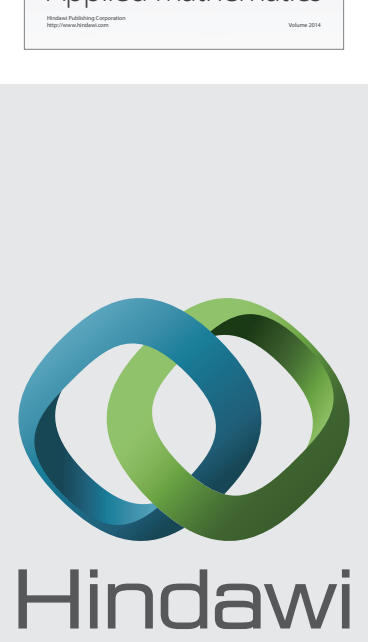

Submit your manuscripts at http://www.hindawi.com
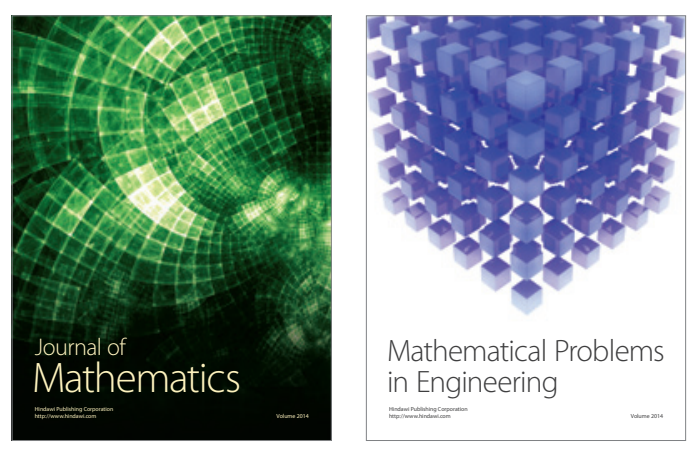

Mathematical Problems in Engineering
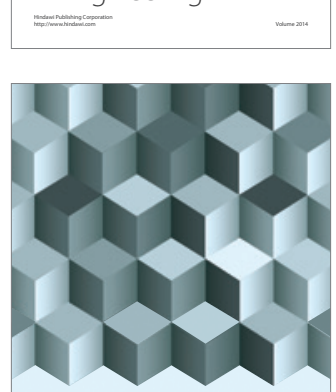

Journal of

Function Spaces
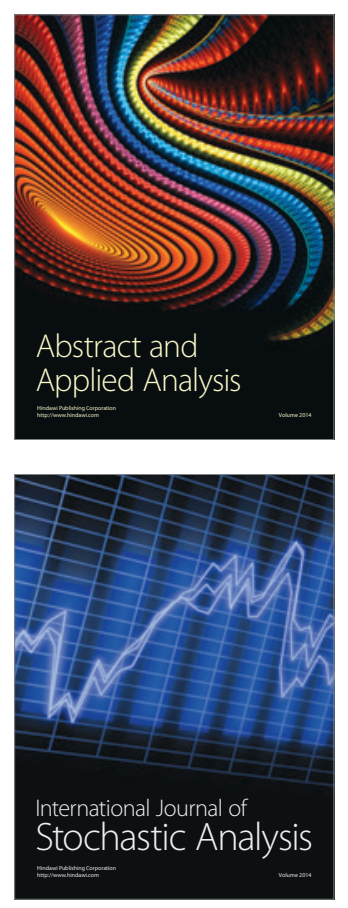

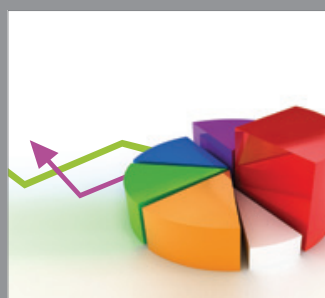

ournal of

Probability and Statistics

Promensencen
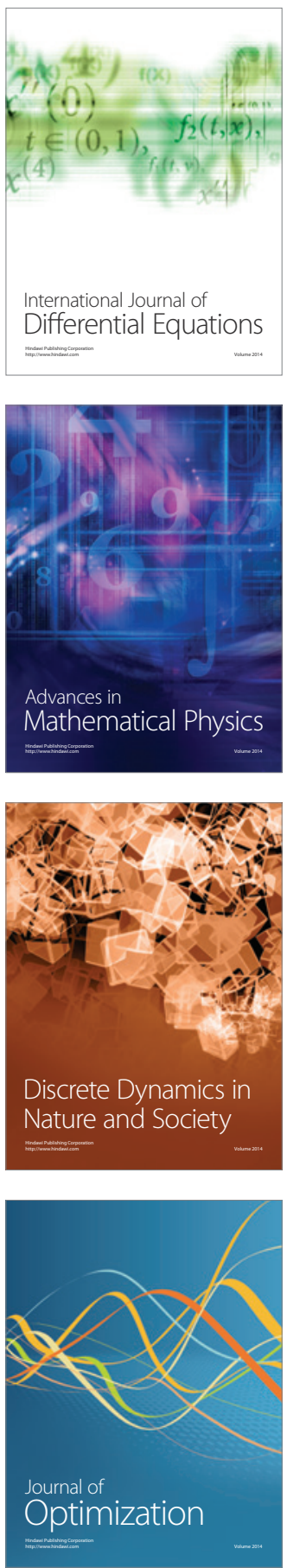